\title{
Repair of a Large Sternal Notch Defect
}

Prasanthi Kandula, MD, Callie Burgin, BS, and Ally-Khan Somani, MD, PhD

Department of Dermatology, Indiana University School of Medicine, Indianapolis, Indiana

This is the author's manuscript of the article published in final edited form as:

Kandula, P., Burgin, C., \& Somani, A.-K. (2019). Repair of a Large Sternal Notch Defect. Dermatologic Surgery, 45(9), 1191-1193. https://doi.org/10.1097/DSS.0000000000001591 
An 84-year-old man presented for treatment of a well-differentiated squamous cell carcinoma (SCC) of the sternal notch (Figure 1A). He had extensive actinic damage, and several other nonmelanoma skin cancers removed in the past. The SCC had been present for several months at the time of Mohs micrographic surgery and measured 1.0 $\times 0.8 \mathrm{~cm}$. Mohs micrographic surgery was performed, and the tumor was removed resulting in a defect measuring $4.2 \times 3.5 \mathrm{~cm}$ extending down to the subcutaneous fat (Figure 1B). How would you reconstruct this defect?

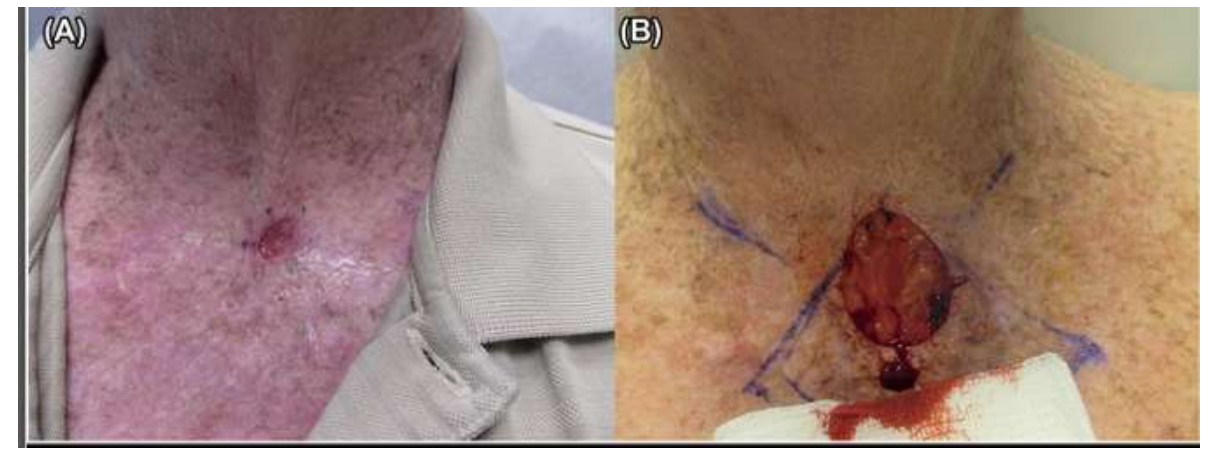

Figure 1. (A) Lesion before Mohs micrographic surgery with tethering of neck skin. (B) Sternal notch defect extending to muscle.

\section{CONSIDERATIONS}

The skin of the sternal notch, base of neck and chest, is very dynamic. The skin here is put under tension constantly with head and shoulder movements. Before removal of the SCC, the skin was tethered and constricted in this area. Our goal for the repair was to allow for movement of the head and upper trunk without feelings of constriction to the patient and avoidance of tethered skin appearance.

Several repair options were discussed with the patient. Secondary intention was not suggested given location, poor cosmetic result, and patient preference. A full-thickness skin graft was also considered but not chosen because of location and size, topographic variation, need for additional surgery, poor cosmetic outcome, and lack of donor site. The clavicle is a common donor site, and the sternal notch and chest are hair-bearing areas. The chance for graft survival is optimized when the graft is laid flat against the defect. The sternal notch and chest are dynamic areas, and graft survival would be difficult here. A transposition and rotation flap were both considered for this defect; the size required and 
secondary tension and distortion from these flaps were considered as a suboptimal choice along with an increased risk for hypertrophic scarring and dehiscence. Similarly, a primary linear closure was also considered but abandoned for the same reasons. The authors describe the repair of the circular sternal notch defect with a novel flap that the authors have named as the "winged" modified keystone flap.

\section{RESOLUTION}

Skin from the inferior portion of the defect was chosen as the leading edge of the advancement flap. A modified keystone was created with a width ratio of 1:1 flap:defect. The flap was positioned over the superior sternum along the C 5 dermatomal segment. It is important for the longitudinal axis of the flap to sit along a dermatomal segment to preserve the cutaneous nerves, veins, and perforators that ensure viability of the flap. The modified keystone was then visually proportioned into thirds. The lateral thirds were completely undermined to create the "wings" and then folded together to account for the increased vertical distance secondary to tension, and a novel "winged" modified keystone flap was created. ${ }^{1}$ A 4-0 polydioxanone suture was then used to secure the tip of the leading edge of the flap to the superior edge of the defect as well as close the lateral secondary defects. A 4-0 prolene running suture was used to approximate all epidermal edges of the flap (Figure 2A-D).

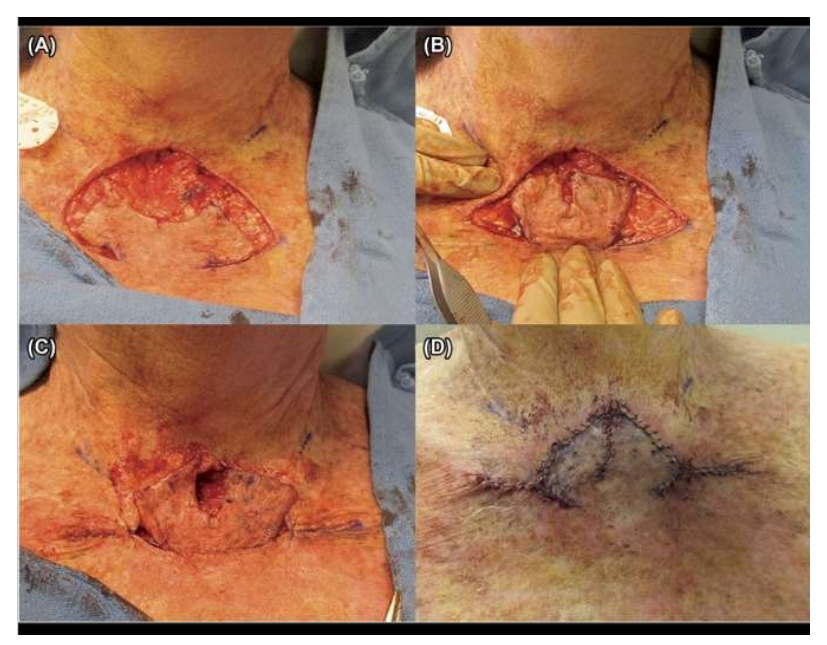

Figure 2.: (A) A modified keystone was designed and incised. (B) The 2 lateral edges or "wings" of the modified keystone flap were completely undermined, raised, and folded together. $(C$ and $D)$ The winged flap was then sutured in place. 
Immediately after the procedure, the flap had good perfusion and the wound was dressed with a pressure bandage. At 1-week follow-up, the flap was healing well, and there was no evidence of necrosis. Sutures were removed at 14 days. Figure 3 shows clinical photographs of the 2-week suture removal and the 7-week follow-up. Overall, patient had an excellent cosmetic result. Tension and tethered appearance were avoided, and dynamic ability was preserved.

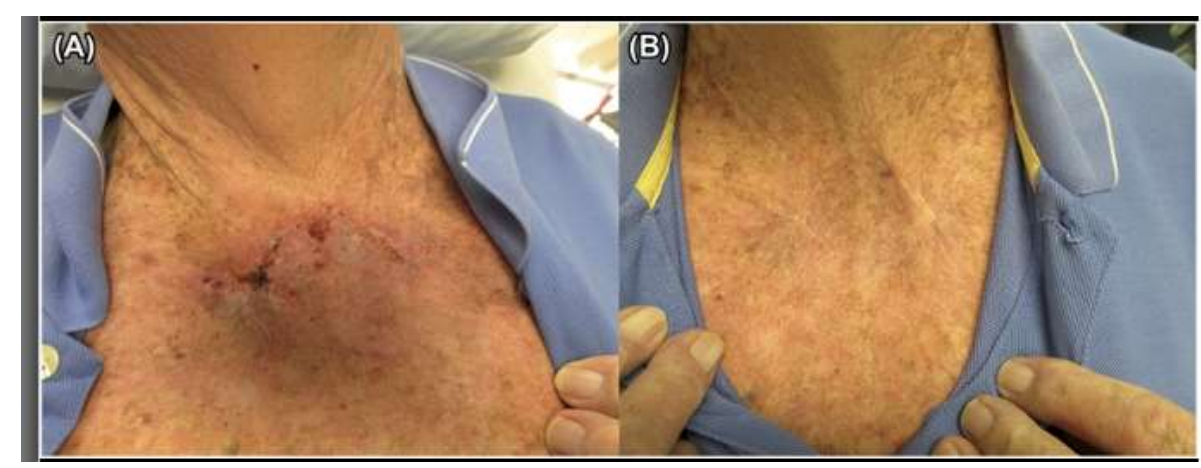

Figure 3.: (A) Suture removal 2 weeks after surgery. (B) Seven weeks after surgery.

The keystone island perforator flap is a multiperforator advancement flap, first described in 2003 by Behana, with a curvilinear trapezoidal shape often used for complex wound reconstruction. Traditionally, a width ratio of 1:1 of flap:defect is used for surgical repairs using keystone island flaps. ${ }^{2}$ The use of the modified keystone island perforator flap, first described by Montcrieff and colleagues, leaves a trailing skin edge of the flap intact for additional vascular supply. This method also speeds closure time by requiring fewer sutures. The keystone and modified keystone flaps are most useful for repair of large surgical defects, typically on the limbs. ${ }^{3}$ Behan and colleagues ${ }^{4}$ also described the use of the keystone island flap for repair of head and neck cancers in elderly patients. The versatility of keystone flaps eliminates the need for split- or full-thickness skin grafts for repair of cutaneous defects unable to be closed with local rotational or advancement flaps. This is of particular benefit in patients at high risk for graft failure such as defects on lower extremities in patients with mobility limitations. ${ }^{5}$

Our "winged" modified keystone flap, which represents a further alteration of the modified keystone island pedicle flap, was chosen for reconstruction of this case given the location and size of the defect. The ability to fold the "wings" of the 2 lateral edges of the modified 
keystone flap affords a facile, tension-free closure along the vertical axis of the defect. The flap can be used for surgical defects in locations that lack the necessary laxity for primary closure, and in patients who are poor candidates for transposition or rotation flaps. Our flap allows coverage of a greater vertical distance while minimizing tension on the trailing intact skin edge and eliminating the need for creation of wider flaps. The adaptation of this novel "winged" modified keystone flap extends the versatility of the existing keystone and modified keystone flaps and should be considered as a viable simple surgical reconstructive option.

\section{CONUNDRUM KEYS}

(1) The novel winged modified keystone island flap expands the available reconstruction options for patients undergoing Mohs surgery.

(2) Creating a winged modified keystone island flap allows for coverage of greater vertical distance from the leading edge of the flap while reducing tension on the trailing skin BRIDGE.

\section{REFERENCES}

1. Behan F. The keystone design perforator island flap in reconstructive surgery. ANZ J Surg 2003;73:112-20.

2. Mohan AT, Rammos CK, Adhavan AA, Martinez J, et al. Evolving concepts of keystone perforator island flaps (KPIF): principles of perforator anatomy, design modifications, and extended clinical applications. Plast Reconstr Surg 2016;137:1909-20.

3. Moncrieff MD, Bowen F, Thompson JF, Saw RP, et al. Keystone flap reconstruction of primary melanoma excision defects of the leg-the end of the skin graft? Ann Surg Oncol 2008;15:2867-73.

4. Behan F, Sizeland A, Gilmour F, Hui A, et al. Use of the keystone island flap for advanced head and neck cancer in the elderly-a principle of amelioration. J Plast Reconstr Aesthet Surg 2010;63:739-45.

5. Stone JP, Webb C, McKinnon JG, Dawes JC, et al. Avoiding skin grafts: the keystone flap in cutaneous defects. Plast Reconstr Surg 2015;136:404-8. 